\title{
pH-Sensitive Dye-Based Nanobioplatform for Colorimetric Detection of Heterogeneous Circulating Tumor Cells
}

Xiuli Wang, ${ }^{1}$ Shasha Cheng, ${ }^{1}$ Xinjun Wang, ${ }^{2,3}$ Liran Wei, ${ }^{2}$ Qianqian Kong, ${ }^{1}$ Mingqiang Ye, ${ }^{1}$ Xianzhu Luo, ${ }^{1}$ Jiao Xu, ${ }^{1}$ Cuiling Zhang ${ }^{1,}{ }^{*}$ and Yuezhong Xian ${ }^{1, *}$

${ }^{1}$ Shanghai Engineering Research Center of Molecular Therapeutics and New Drug Development, Department of Chemistry, School of Chemistry and Molecular Engineering, East China Normal University, Shanghai 200241, China

${ }^{2}$ Shanghai Zhangjiang Institute of Medical Innovation, Shanghai 201204, China

${ }^{3}$ Translational Medical Center for Stem Cell Therapy and Institute for Regenerative Medicine, Shanghai East Hospital, School of Life Sciences and Technology, Tongji University, Shanghai, 200092, China.

*Corresponding Authors

E-mail: clzhang@chem.ecnu.edu.cn,

E-mail: yzxian@chem.ecnu.edu.cn. 


\section{Table of Contents}

$\begin{array}{ll}\text { 1. Reagents } & \text { S3 }\end{array}$

2. Instruments and characterization $\quad \mathrm{S} 4$

3. UV-vis spectra of AuNP and aptamer-AuNP S5

4. The zeta potential for AuNP and aptamer-AuNP S6

5. The zeta potential of PAA-MoS 2 NFs

6. UV-vis spectra of the prepared nanomaterials $\quad$ S8

7. High-resolution XPS spectra of C 1s and P 2p S9

8. The absorbance spectra and standard absorbance curves of dyes $\quad$ S10

9. The cell images of multivalent aptamer-AuNP nanoconjugates and monovalent $\begin{array}{ll}\text { aptamer } & \text { S11 }\end{array}$

10. UV-vis spectra of lysed blood samples from 7 breast cancer patients $\quad$ S12

11. UV-vis spectra of lysed blood samples from 3 cervical cancer patients $\quad$ S13

12. Comparison of different methods for the detection of heterogeneous CTCs $\quad$ S14

$\begin{array}{ll}\text { 13. References } & \text { S15 }\end{array}$ 
Reagents. $\left(\mathrm{NH}_{4}\right)_{6} \mathrm{Mo}_{7} \mathrm{O}_{24} \cdot 4 \mathrm{H}_{2} \mathrm{O}(\geq 99.0 \%, \mathrm{AR})$, thioacetamide (TAA, $\geq 99.0 \%$, AR) and trisodium citrate dihydrate were obtained from Sinopharm Chemical Reagent Co., Ltd. (Shanghai, China). N-2-hydroxyethylpiperazine-N'-2-ethanesulfonic acid (HEPES, $\geq 99.5 \%$ ), PAA (MW $\approx 2000)$ (Shanghai, China) and gold chloride solution were purchased from Aladdin Reagent Company (Shanghai, China). Streptavidin (SA) was bought from Yuanye Biological Technology Co., Ltd. (Shanghai, China). Bovine serum albumin (BSA, >98.0\%) was acquired from Life Sciences Research Products \& Services (Shanghai, China). N-(3-(Dimethylamino)propyl)-N'-ethylcarbodiimide hydrochloride (EDC) was obtained from Alfa Aesar Co., Ltd. (Beijing, China). AntiCK8-Alexa Fluor 594 and anti-CD45-FITC were purchased from Youningwei Biotechnology Co., Ltd. (Beijing, China). Curcumin (CUR), thymolphthalein (TP) and N-hydroxysuccinimide (NHS) were purchased from Macklin Biochemical Technology Co., Ltd. (Shanghai, China). Red blood cell lysate was purchased from Solaibao Technology Co., Ltd. (Beijing, China). All the other reagents were analytical grade and utilized directly without further treatments. Ultrapure water was used throughout the experiments. The aptamers were purchased from Sangon Biotech Co., Ltd. (Shanghai, China) and the sequences for corresponding aptamers were listed as followings.

Aptamer C-9S: 5'-NH2-C6-TTT TTT TTT ATTCAA TTA TCG TCC GCT TTA GTG TAA TGG TCC GTC CCG CAG TGA ATAA-3' \& 5'-SH-C6-TTTTTTTTTT TTA TTC AAT TAT CGT CCG CTT TAG TGT AAT GGT CCG TCC CGC AGT GAA TAAbiotin-3'.

Aptamer SYL3C: 5'-NH2-C6-TTTTTTTTTT CAC TAC AGA GGT TGC GTC TGT 
CCC ACG TTG TCA TGG GGG GTT GGC CTG-3' \& 5'-SH-C6-TTTTTTTTTT CAC

TAC AGA GGT TGC GTC TGT CCC ACG TTG TCA TGG GGG GTT GGC CTGbiotin-3'.

Instruments and Characterization. Transmission electron microscope (TEM) imagings were conducted on an HT-7700 Hitachi TEM (Japan). Fourier transform infrared (FTIR) spectrum was obtained on a Thermo Fisher Scientific Nicolet iS50 FTIR spectrometer (USA). UV-vis measurements were carried out by using a UV-2550 spectrophotometer (Japan). X-ray diffraction (XRD) was harvested from an Smartlab SE X-ray powder diffractometer (Japan). The fluorescence images were measured on a Ti-S/L100 inverted microscope (Nikon). Electrochemical impedance spectra (EIS) were performed on a Metrohm Autolab PGSTAT302N electrochemical station (Switzerland). The Zeta potential and dynamic light scattering (DLS) for particle size analysis were measured with a Nano particle size and Zeta potential analyzer (NANO ZS3600). The absorption spectra obtained in the 96-well plates were identified by a Multimode Microplate Reader (TECAN, Switzerland). All the digital photos were taken with a smartphone (Huawei nova7 pro, China). 


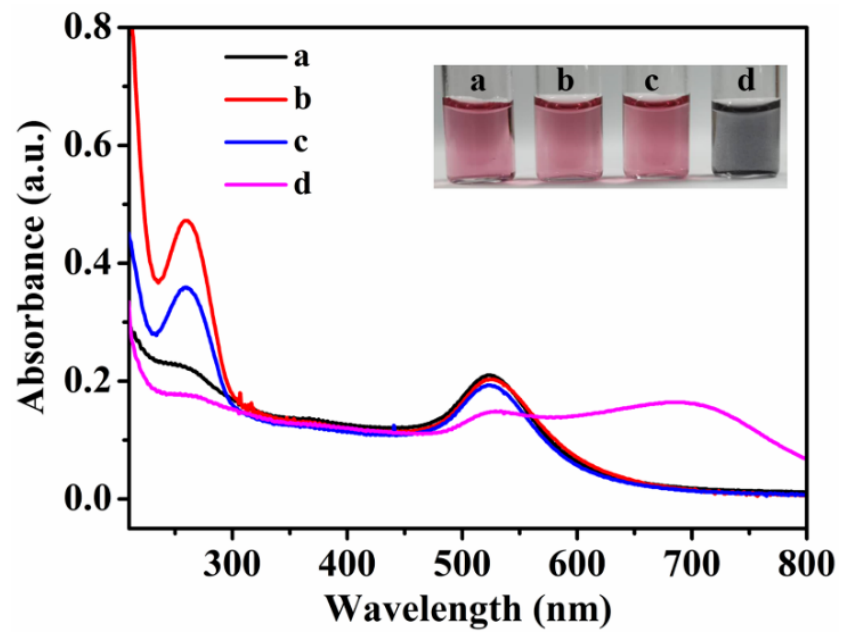

Figure S1. UV-vis spectra and digital photos (inset) of AuNP before freeze-thaw (black line, inset a), the mixture of aptamer and AuNP before freeze-thaw (red line, inset b), aptamer-AuNP after freeze-thaw (blue line, inset c) and colloidal of AuNP after freezethaw (purple line, inset d). 


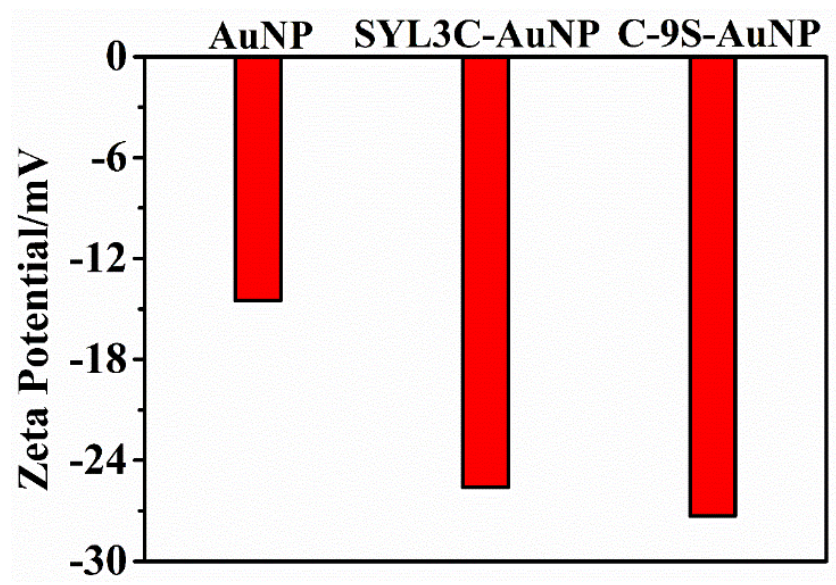

Figure S2. Zeta potential for AuNP, SYL3C-AuNP and C-9S-AuNP. 


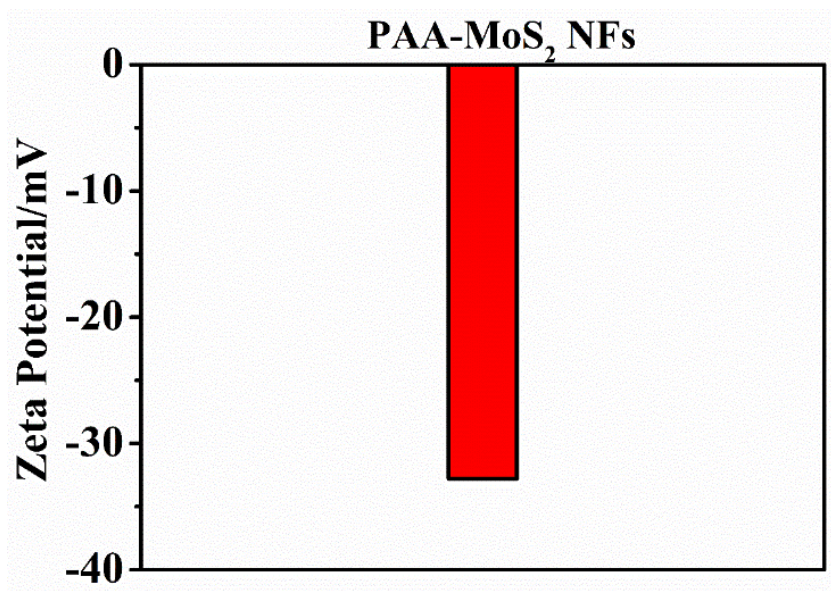

Figure S3. Zeta potential of PAA-MoS 2 NFs. 

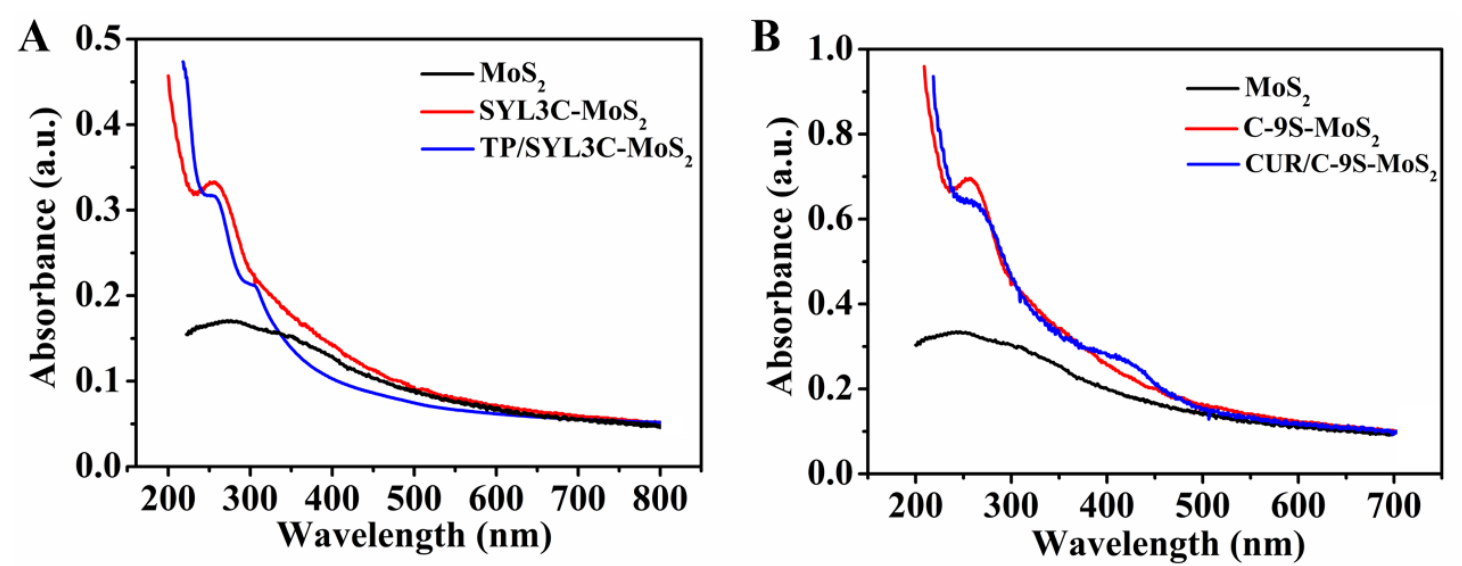

Figure S4. (A) UV-vis spectra of $\mathrm{MoS}_{2}$, SYL3C-MoS 2 and TP/SYL3C-MoS 2 in neutral solution (pH 7.0). (B) UV-vis spectra of $\mathrm{MoS}_{2}, \mathrm{C}-9 \mathrm{~S}-\mathrm{MoS}_{2}$ and CUR/C-9S-MoS 2 in neutral solution ( $\mathrm{pH} 7.0)$. 

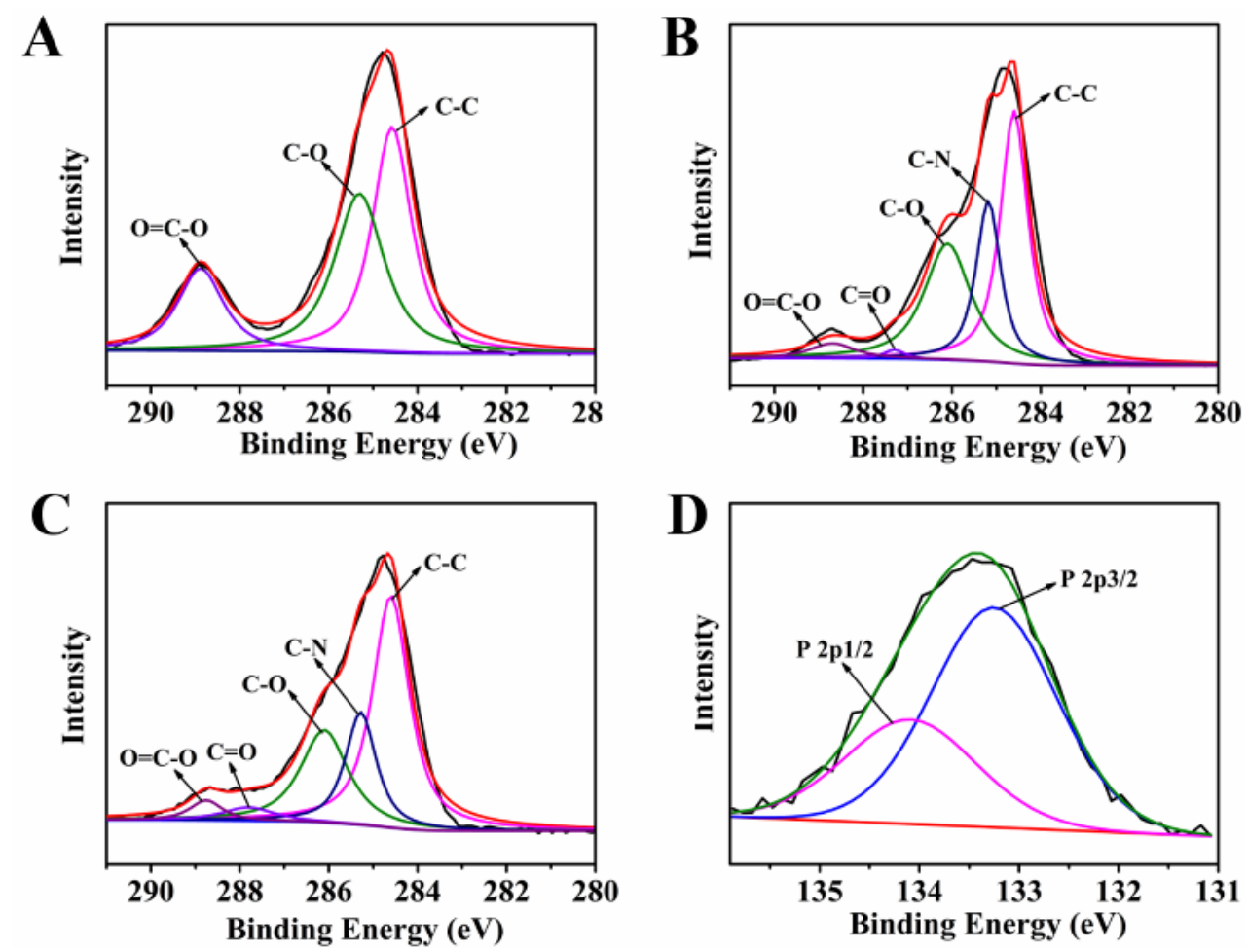

Figure S5. High-resolution XPS spectra of C 1s for (A) PAA-MoS 2 , (B) aptamer/MoS2, (C) dye/aptamer/MoS 2 and (D) high-resolution XPS spectra of P 2p. 

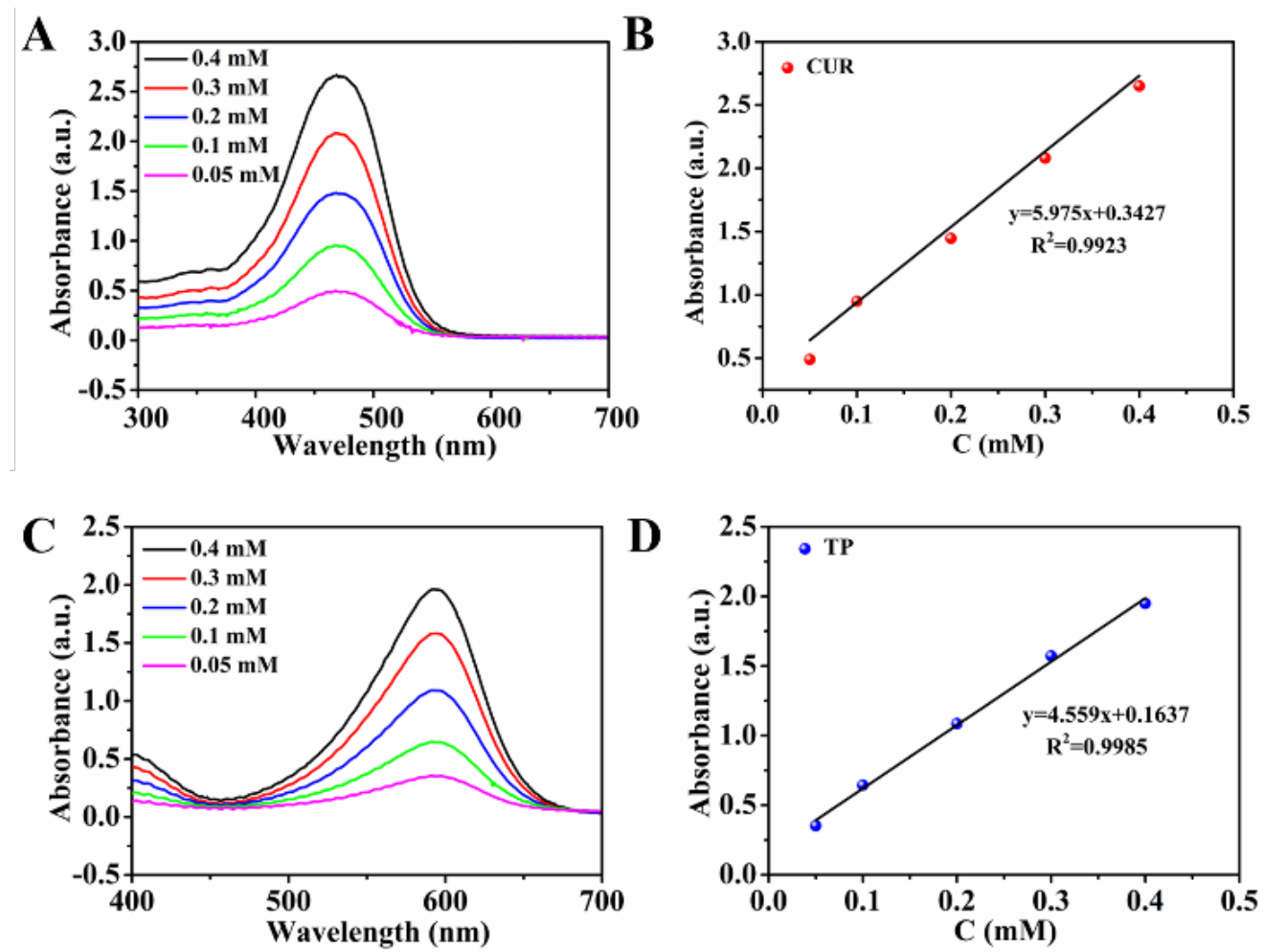

Figure S6. The absorbance spectra of different concentrations of CUR (A) and TP (C), and their corresponding standard absorbance curves for CUR at $470 \mathrm{~nm}$ (B) and TP at $590 \mathrm{~nm}$ (D). $20 \mu \mathrm{L}$ of TP and CUR with different concentration (0.05, 0.1, 0.2, 0.3 and $0.4 \mathrm{mM}$ ) were incubated with $100 \mu \mathrm{L} \mathrm{NaOH}(\mathrm{pH} 12.5)$ for $80 \mathrm{~s}$, and then the UV-vis spectra were recorded. 


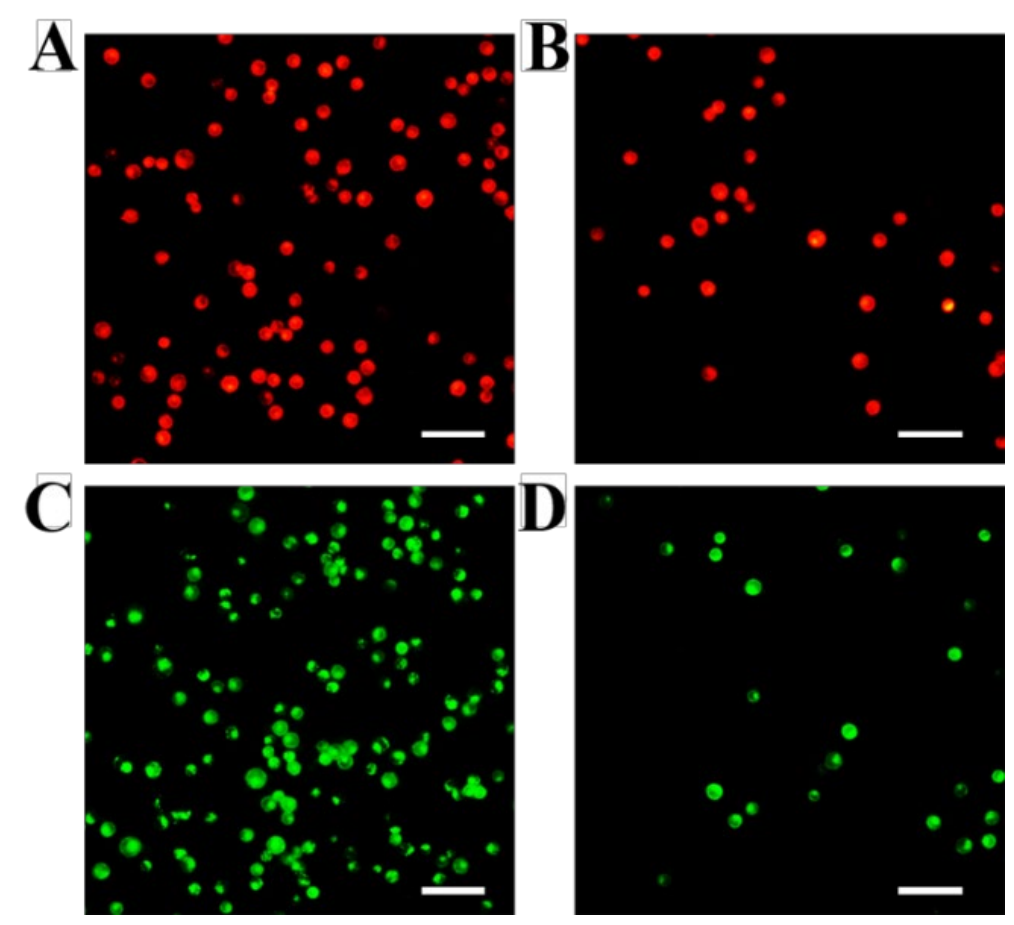

Figure S7. Representative images of the target MCF-7 cells (red) and HeLa cells (green) that were captured in 96-well plates by multivalent aptamer-AuNP nanoconjugates (A, C) and monovalent aptamer (B, D). Scale bar $=100 \mu \mathrm{m}$. 

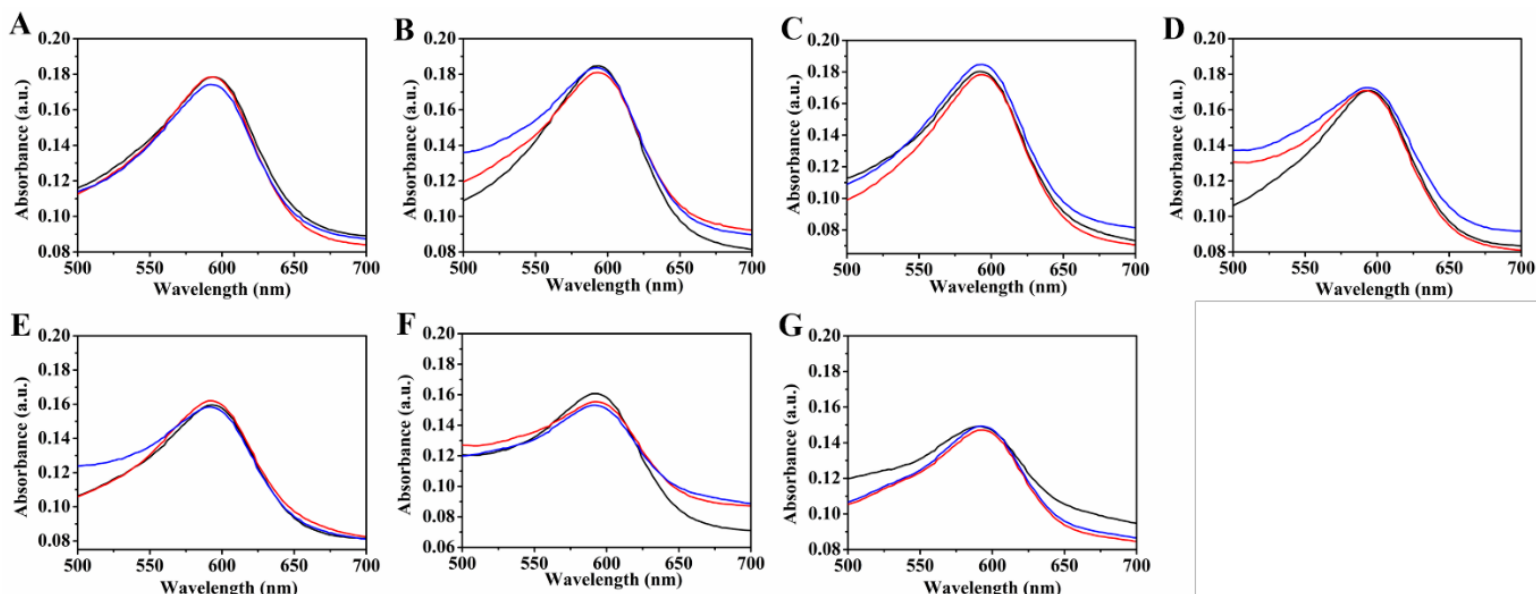

Figure S8. (A-G) UV-vis spectra of lysed blood samples from 7 breast cancer patients and each blood sample was repeated three times (black line, red line and blue line). 

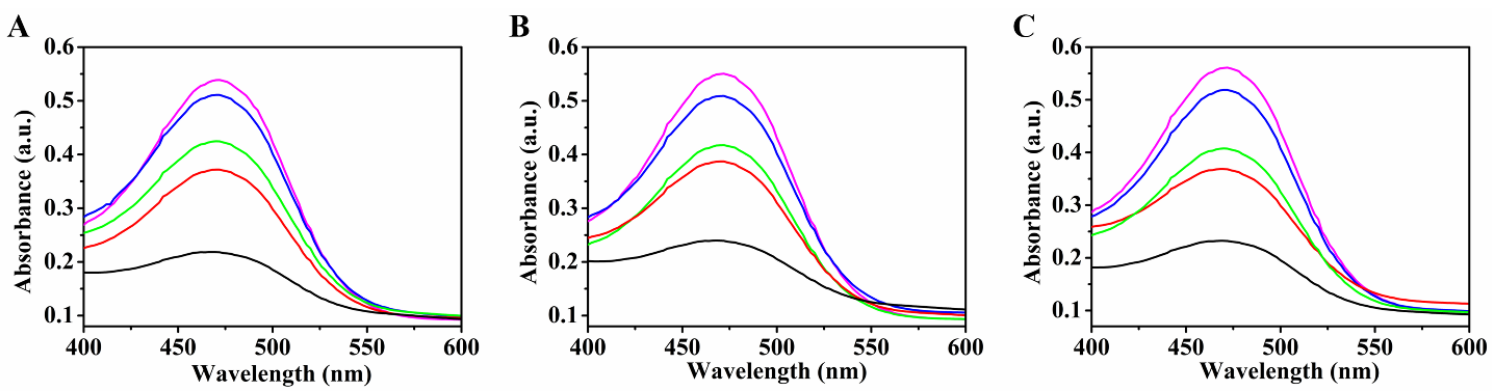

Figure S9. (A-C) UV-vis spectra of HeLa cells spiked in lysed blood samples from 3 cervical cancer patients: 0 cells $\mathrm{mL}^{-1}$ (black line), 20 cells $\mathrm{mL}^{-1}$ (red line), 50 cells $\mathrm{mL}^{-}$

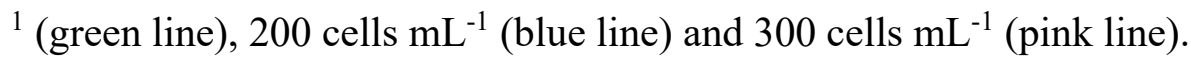


Table S1. Comparison of different methods for the detection of heterogeneous CTCs.

\begin{tabular}{|c|c|c|c|c|c|}
\hline Method & Materials & $\begin{array}{l}\text { Target Cells \& } \\
\text { Capture rate }\end{array}$ & $\begin{array}{l}\text { Linear Range } \\
\text { (cells } \mathrm{mL}^{-1} \text { ) }\end{array}$ & $\begin{array}{l}\text { Detection Limit } \\
\left(\text { cells } \mathrm{mL}^{-1}\right)\end{array}$ & Ref \\
\hline Fluorescence & $\mathrm{Fe}_{3} \mathrm{O}_{4}-\mathrm{MVs}-\mathrm{DBCO}$ & $\begin{array}{l}\text { above } 84 \% \text { and } 82 \% \\
\text { for A549 and HepG2 }\end{array}$ & $5-200$ & 5 and 5 & 1 \\
\hline Fluorescence & $\begin{array}{l}\text { PLGA nanofibers } \\
\text { substrate }\end{array}$ & $\begin{array}{l}\text { over } 70 \% \text { for MCF-7 } \\
\text { and GIST } 882\end{array}$ & $10-200$ & 10 and 10 & 2 \\
\hline Electrochemistry & $\mathrm{AuNPs}-\mathrm{Fe}_{3} \mathrm{O}_{4}-\mathrm{GS}$ & $\begin{array}{c}\text { detection Ramos and } \\
\text { CCRF-CEM }\end{array}$ & $5-500$ & 4 and 3 & 3 \\
\hline $\begin{array}{l}\text { Gradient } \\
\text { Magnetic } \\
\text { Separation }\end{array}$ & BFMNPs-Ab & $\begin{array}{l}\text { about } \quad 90 \% \text { for } \\
\text { BT474, LNCaP and } \\
\text { MDA-MB-231 }\end{array}$ & $1-1000$ & 1 and 1 & 4 \\
\hline Microchip & $\begin{array}{c}\text { G-OEMBNs-EGFR+Y- } \\
\text { OEMBNs-HER2+R- } \\
\text { OEMBNs-EpCAM }\end{array}$ & $\begin{array}{c}\text { over } 91.6 \% \text { for } \\
\text { MCF-7, HeLa and } \\
\text { SK-BR-3 }\end{array}$ & $15-1000$ & -- & 5 \\
\hline Colorimetry & $\begin{array}{c}\text { TP/SYL3C- } \\
\mathrm{MoS}_{2}+\mathrm{CUR} / \mathrm{C}-9 \mathrm{~S}-\mathrm{MoS}_{2}\end{array}$ & $\begin{array}{c}\text { over } 90 \% \text { for MCF-7 } \\
\text { and HeLa }\end{array}$ & $5-10000$ & 2 and 4 & $\begin{array}{l}\text { This } \\
\text { Work }\end{array}$ \\
\hline
\end{tabular}




\section{References:}

1. Kang, K.; Zhou, X. X.; Zhang, Y. J.; Zhu, N. H.; Li, G. H.; Yi, Q. Y.; Wu, Y. Cellreleased magnetic vesicles capturing metabolic labeled rare circulating tumor cells based on bioorthogonal chemistry. Small 2021, DOI: 10.1002/smll.202007796.

2. Liu, H.; Wang, Z. L.; Chen, C. C.; Ding, P.; Sun, N.; Pei, R. J. Dual-antibody modified PLGA nanofibers for specific capture of epithelial and mesenchymal CTCs. Colloids Surf. B 2019, 181, 143-148.

3. Dou, B. T.; Xu, L.; Jiang, B. Y.; Yuan, R.; Xiang, Y. Aptamer-functionalized and gold nanoparticle array-decorated magnetic graphene nanosheets enable multiplexed and sensitive electrochemical detection of rare circulating tumor cells in whole blood. Anal. Chem. 2019, 91, 10792-10799.

4. Liao, Z. Y.; Han, L.; Li, Q. J.; Li, L. Y.; Liu, Y.; Song, Y.; Tan, W. H.; Song, E. Q. Gradient magnetic separation and fluorescent imaging based heterogeneous circulating tumor cell subpopulations assay with biomimetic multifunctional nanoprobes. $A d v$. Funct. Mater. 2021, DOI: 10.1002/adfm.202009937.

5. Wu, L. L.; Zhang, Z. L.; Tang, M.; Zhu, D. L.; Dong, X. J.; Hu, J.; Qi, C. B.; Tang, H. W.; Pang, D. W. Spectrally combined encoding for profiling heterogeneous circulating tumor cells using a multifunctional nanosphere-mediated microfluidic platform. Angew. Chem. Int. Ed. 2020, 132, 11336-11340. 